Revue d'histoire de l'Amérique française

ZRS REVUE D.HISTOIRE DE L'AMÉRIQUE FRANÇAISE

\title{
Un projet de musée national à Québec à l'époque du peintre Joseph Légaré (1833-1853)
}

\section{John R. Porter}

Volume 31, numéro 1, juin 1977

URI : https://id.erudit.org/iderudit/303583ar

DOI : https://doi.org/10.7202/303583ar

Aller au sommaire du numéro

Éditeur(s)

Institut d'histoire de l'Amérique française

ISSN

0035-2357 (imprimé)

1492-1383 (numérique)

Découvrir la revue

Citer cette note

Porter, J. R. (1977). Un projet de musée national à Québec à l'époque du peintre Joseph Légaré (1833-1853). Revue d'histoire de l'Amérique française, 31(1),

75-82. https://doi.org/10.7202/303583ar d'utilisation que vous pouvez consulter en ligne.

https://apropos.erudit.org/fr/usagers/politique-dutilisation/ 


\section{UN PROJET DE MUSÉE NATIONAL À QUÉBEC À L'ÉPOQUE DU PEINTRE JOSEPH LÉGARÉ}

(1833-1853)

JOHN R. PORTER

Galerie nationale du Canada

L'ouverture du Musée du Québec eut lieu à Québec en 1933, onze ans après la sanction de la Loi des musées de la province. Ce fut alors l'aboutissement de multiples projets dont le plus ancien remontait à l'époque du peintre Joseph Légaré (1795-1855), l'un des premiers promoteurs d'un musée national à Québec. La genèse et le contenu de ce projet nous révèlent le caractère somme toute avantgardiste de certains membres de l'élite intellectuelle de Québec au milieu du XIX ${ }^{\mathrm{e}}$ siècle.

Avant que ne soit mis de l'avant le projet spécifique de musée national, un certain nombre d'initiatives privées lui ont servi de prémisses. Avec le recul des ans, nous sommes plus en mesure de saisir l'importance d'initiatives comme le "Musée Chasseur», "La Galerie de Peinture de Québec» et le projet d'un «Institut Vattemare».

C'est en 1824 que Pierre Chasseur fonda à Québec un musée d'histoire naturelle, le premier musée au Canada. Dans un article paru en 1826 dans la Gazette de Québec' et repris la même année dans $L a$ Bibliothèque canadienne, le rédacteur faisait l'éloge de Chasseur et de sa «collection qui prouve les miracles que peuvent faire le génie, l'industrie et la persévérance». Cette collection comprenait environ trois cents sujets (animaux) autour desquels Chasseur avait rassemblé «tout ce qui tend à [les] caractériser, de manière à nous donner tout à la fois en quelque sorte l'histoire et les habitudes [des animaux] en vue». À la fin de son article, l'auteur disait regretter que Chasseur ne dispose pas encore de locaux suffisamment spacieux pour mettre

RHAF, vol. 31, n 1 (juin 1977) 
en valeur sa collection ${ }^{1}$. L'année suivante, Chasseur remerciait ceux qui avaient appuyé financièrement son musée jusque-là. Par la même occasion, il offrait aux citoyens de Québec le libre accès de son établissement au cours des cinq années suivantes pour la modique somme de dix shillings. Par cette souscription, il disait espérer construire un édifice additionnel susceptible d'accueillir adéquatement une collection alors en plein développement ${ }^{2}$.

Dans le domaine des beaux-arts, Joseph Légaré fut le pendant de son ami Pierre Chasseur. En effet, c'est à lui que revient le mérite d'avoir créé la première galerie de peinture de Québec et de tout le pays. Le 22 novembre 1833, Légaré faisait paraitre l'annonce suivante:

Le Soussigné ayant réuni à sa maison neuve, située rue St. Angèle, toute sa collection de peintures et gravures, dont la plus grande partie est de la main des premiers peintres d'Europe et étant la plus riche collection de ce genre qui ait jamais paru en Canada, prend la liberté d'informer ses amis et le public en générial quiu'elle est exposóé à là vủe des amâteurs tous les jours de la semaine entre dix heures du matin et quatre heures du soir, excepté les fêtes et dimanche. $[\ldots]^{3}$.

Cinq ans plus tard, Légaré s'associait à l'avocat Thomas Amiot et ils ouvraient «La Galerie de Peinture de Québec» aux étages supérieurs d'un édifice que venait de faire élever ce dernier du côté nord de la place du marché à la haute-ville. Plusieurs personnes saisirent l'importance de cet événement pour le développement des beaux-arts et le situèrent dans une perspective nationaliste:

Nous désirons ardemment de voir encourager ce premier effort pour répandre le goût des beaux-arts, et d'autant plus que l'honneur en est dû tout entier à des natifs du pays, descendants des premiers colons français ${ }^{4}$.

Un pareil établissement est un honneur, une gloire pour le pays, pour la ville qui le possède ${ }^{5}$.

1 La Bibliothèque canadienne, III, $\mathrm{n}^{\circ} 1$ (juin 1826): 35.

2 The Quebec Mercury, 17 mars 1827, 130. Ancien sculpteur et doreur, Chasseur vendit sa collection au gouvernement en 1835. En 1837-1838, il fut l'un des Patriotes de la région de Québeo. Il mourut dans cette ville le 21 mai 1842. Voir Pierre-Georges Roy, «Le premier musée au Canada», dans le Bulletin des recherches historiques, XXXII, $\mathrm{n}^{\circ} 11$ (novembre 1926): 641-646.

3 Le Canadien, 22 novembre 1833, 3.

4 La Gazette de Québec, citée dans Le Canadien, 27 juillet 1838, 3.

5 Le Canadien, 25 juin 1838, 3. 
En 1840, la ville de Québec accueillit un visiteur de prestige en la personne du français Alexandre Vattemare (1796-1864) dont le projet d'institut allait mériter l'appui de Légaré. Homme pour le moins original, Vattemare avait créé un système d'échange international de spécimens doubles dans les domaines des arts, des sciences naturelles, des ouvrages scientifiques et littéraires ${ }^{6}$. Lors de ses visites à Montréal puis à Québec, Vattemare ajouta à son système d'échange un projet d'institut de conception grandiose. Cet institut était appelé à réunir les sociétés déjà existantes. Dans le cas de Québec, il s'agissait de la «Société Littéraire et Historique de Québec», de l' «Institut des Artisans» et de la «Bibliothèque de Québec». On suggéra alors que l'ancien collège des Jésuites soit affecté aux fins de l'Institut Vattemare. Le 2 mars 1841, lors d'une grande réunion d'adieux présidée par le maire René-Édouard Caron et qui attira environ trois mille personnes, Vattemare précisa ses vues quant à l'Institut qu'il souhaitait voir s'établir à Québec:

Je viens donc à présent vous demander au nom de ceux de vos compatriotes qui sont encore dans l'ignorance, au nom de tout ce qui vous est cher, au nom de vos enfants, de votre patrie, une sincère et constante coopération pour la création d'une institution du genre de celle que je propose et qui devra comprendre bibliothèque, cabinet d'histoire naturelle, musée d'arts, collection d'objets d'intérêt général, académie de dessin et de peinture, salle de lecture et de cours publics, enfin tout ce qui peut aider au développement du génie, du goût et de l'intelligence.

Malgré l'étendue et les réclusses [richesses] que devra contenir cet établissement, sa formation en est simple, facile et peu coûteuse; le moindre examen vous en convaincra. Du moment où un pareil institut sera ouvert et modestement doté de manière à lui assurer sa permanence; qu'il sera confié à des personnes habiles qui s'y consacreront entièrement et attacheront de la gloire à son aggrandissement, des contributions volontaires, telles que tableaux, médailles, livres, gravures, curiosité fossiles, coquilles, minéraux, modèles d'inventions, vieilleries de tout genre, etc. [;] tout trouvera sa place dans un semblable établissement $(\ldots)^{7}$

6 Élisabeth Revai, Alexandre Vattemare, trait d'union entre deux mondes (Montréal-Paris, Bellarmin-Desclée de Brouwer, 1975), 221 pages.

7 Le Canadien, 5 mars 1841 , 2. La visite de Vattemare suscita un grand enthousiasme dans le public, comme en témoignent les journaux. Voir notamment Le Canadien du 8 février au 17 mars 1841. 
Dans une envolée pathétique, l'illustre Vattemare fit appel à la fierté et à la grandeur d'âme de ses auditeurs:

Chacun de vous, j'en suis sûr rivalisera de zèle pour apporter sa pierre à l'édifice qui va s'élever et que chacun de vous voudra dire: ce monument qui fait l'honneur et la prospérité de notre pays, $\mathrm{j}$ 'ai contribué à son érection; ce sera votre enfant, votre joie; vous l'entretiendrez, le soignerez, le visiterez et le pauvre qui apportera son denier le regardera avec autant d'orgueil que son frère, homme riche et puissant, qui en aura donné mille, car vos offrandes seront proportionnelles à vos moyens ${ }^{8}$.

Un comité général fut mis sur pied pour travailler à la réalisation de l'Institut Vattemare. L'architecte Thomas Baillairgé, l'historien François-Xavier Garneau, le sculpteur François-Xavier Leprohon de même que les peintres Antoine Plamondon et Joseph Légaré en faisaient partie ${ }^{9}$. Malheureusement leurs espérances et celles de Vattemare ne devaient pas se réaliser, l'unanimité créée lors du passage de ce dernier n'ayant pas tardé à se briser après son départ ${ }^{10}$.

Il semble toutefois que certaines des idées mises de l'avant par Vattemare aient eu une incidence sur la formulation du premier projet de "galerie nationale» à Québec, en 1845. Le 20 février, onze citoyens de Québec - dont Joseph Légaré, Napoléon Aubin, rédacteur du journal Le Castor, le notaire Archibald Campbell ${ }^{11}$, le juge Édouard Bacquet ${ }^{12}$ et le Dr William Sewell - adressaient la lettre suivante à Édouard Glackmeyer, président du Conseil de ville de Québec:

Monsieur,

Voyant que la corporation de la cité de Québec vient d'accepter

l'offre qui lui a été faite par le gouvernement provincial de la

8 Loc. cit.

$9 \quad$ Ibid., 12 mars $1841,2$.

10 Le Canadien, 6 décembre $1847,2$.

11 Dans Le Castor du 4 juin 1844 , le notaire Campbell offre des tableaux en vente à son bureau de la rue St-Paul. Les titres de certains d'entre eux recoupent ceux d'œuvres de Légaré. L'annonce fut reprise une dizaine de fois entre le 13 juin et le 17 octobre 1844 .

12 Le juge Bacquet possédait une importante collection de tableaux et de gravures. Il était propriétaire d'un certain nombre de tableaux en société avec Légaré. Voir Archives nationales du Québec, Québec, Greffe du notaire Octave Chavigny de la Chevrotière, $n^{\circ} 383,18-22$ avril et 7 mai 1853, "Inventaire des biens dépendant de la succession de feu l'Honorable Juge Bacquet». 
disposition des édifices publics de cette ville, nous prenons la liberté d'attirer l'attention des membres du conseil sur la convenance et l'utilité de consacrer une des salles de ces vastes bâtisses à l'avantage général du public en y permettant l'exposition des tableaux, gravures, livres, produits des beaux-arts que des particuliers voudraient y déposer. Un tel local, qui servirait au besoin d'école de dessin et de peinture, serait ouvert au public à de certaines heures, et les artistes pourraient y venir travailler comme ils le font dans les galeries nationales des autres pays plus avancés que le nôtre sous ce rapport.

Nous sommes induits à faire ces suggestions au Conseil par la persuasion que l'Exécutif, en confiant aux autorités municipales la disposition et la garde des édifices publics à des conditions aussi libérales, a cru pouvoir par là contribuer plus directement à l'avantage général et faciliter l'admission des demandes qui lui étaient faites par des sociétés et des corps savants pour l'occupation d'appartements auxquels ils pensaient en cette qualité avoir quelques droits.

Nous ne voyons pas qu'il soit nécessaire de faire remarquer que toutes les autres villes, même moins importantes que la nôtre, doivent à des administrations éclairées des institutions de ce genre. Si l'on en est privé à Québec, on ne peut l'attribuer au défaut d'éléments convenables, car peu de villes sur ce continent possèdent plus de tableaux précieux, de gravures anciennes, que l'on ne pourrait en réunir ici. Pourquoi ne profiterionsnous pas de l'occasion favorable qui nous est offerte, qui ne se rencontrera peut-être plus, de commencer une institution dont on sentirait bien vite l'agrément et l'utilité, qui entretiendrait, cultiverait le goût des beaux-arts et en faciliterait l'étude à la jeunesse? pourquoi ne chercherions-nous point à augmenter encore l'attrait que les étrangers trouvent à visiter notre ville, en fournissant à leur curiosité les objets d'arts qui demeurent ignorés ou dispersés aujourd'hui entre les mains de particuliers qui en jouissent seuls, sans aucun résultat pour le pays? [... $]^{13}$

Il ne semble pas que cet habile plaidoyer ait produit les effets escomptés. En effet, trois ans plus tard, Légaré exposait, mais de façon temporaire, quelques-unes de ses œuvres personnelles ainsi que certains tableaux de sa collection dans l'un des appartements de la Chambre d'Assemblée à Québec ${ }^{14}$. Après avoir souligné la qualité de

13 Le Castor, 24 février 1845, 2.

14 Le Canadien, 29 septembre 1848,2 et 3 . Une annonce parue ce jour-là fut reprise onze fois jusqu'au 27 octobre. 
ces œuvres, un journaliste du journal Le Canadien reprocha au public son indifférence face à l'exposition. Tirant ses conclusions, il en rejeta le blâme sur le gouvernement qui, selon lui, négligeait de fournir une salle convenable qui fut suffisamment spacieuse pour abriter toute la collection de Légaré :

Mais pourquoi s'étonner de cette indifférence quand l'exemple en vient de si haut? Si le gouvernement ne protège point les arts, il est vain de compter sur l'appui des particuliers.

Quand je dirai que ce même M. Légaré possède chez lui plus de cent cinquante tableaux originaux (reste de ceux qu'emporta de France M. Desjardins, exilé en 1793), égaux, sinon supérieurs en mérite aux deux que j'ai déjà nommés; que faute d'une salle pour les exposer, ces chefs-d'œuvre restent dos-à-dos, à terre dans un grenier; que, malgré ses pressantes sollicitations, on n'a point voulu, on ne veut point encore permettre à $M$. Légaré de rendre notre musée (!) un peu plus attrayant en les y exposant, mais qu'on préfère les laisser où ils sont, perdus pour le public et pour les élèves de peinture - l'on s'étonnera peut-être, et ce n'est pourtant que la simple vérité ${ }^{15}$.

Face à cette situation, les propagandistes d'un musée national devaient revenir à la charge cinq ans plus tard. C'est par l'entremise du conseiller William S. Sewell - un des signataires de la lettre de 1848 - secondé par $\mathrm{M}$. Rhéaume, qu'ils soumirent à nouveau leur proposition au Conseil de ville de Québec. Il est intéressant de noter que cette fois ils fondèrent la nécessité d'un musée national au Canada sur l'exemple concret des autres nations. Ceci les amena à suggérer la construction d'un édifice distinct pour l'établissement d'un musée. La proposition du Dr Sewell se lisait comme suit:

1. Que l'avancement des connaissances étant le premier intérêt de tout état, il est du devoir de tous les corps chargés de quelque surveillance concernant le bien public, de contribuer au grand œuvre de la diffusion des lumières dans toutes les classes de la société.

2. Que l'une des marques les plus prononcées et les plus satisfaisantes du siècle présent sur les siècles passés, est l'activité redoublée de la presse, et par le moyen de la multipli-

15 Le Canadien, 13 octobre 1848, 2. En 1852, la Société littéraire et historique de Québec disposait d'une grande salle dans la Chambre d'Assemblée. Cette salle renfermait entre autres un musée d'histoire naturelle - l'ancien Musée Chasseur - et une bibliothèque. Ceux-ci furent détruits lors de l'incendie de l'édifice en 1854. Voir Roy, op. cit.: 645 et The Quebec Mercury, 31 août 1852, 2. 
cation des livres, la diffusion de tous les résultats inappréciables des recherches scientifiques, dont la connaissance était limitée aux seuls savants.

3. Que les lumières ainsi dévoilées à la masse, ne sont qu'imparfaitement transmises sans l'aide des musées qui mettent à la vue de tout le monde des dépôts d'objets qui servent à faire connaître les œuvres de la création et les inventions de l'esprit humain dans tous les siècles.

4. Que de pareils dépôts se trouvent chez toutes les nations éclairées et que le temps est arrivé où il devient à propos pour le Canada de faire un premier pas dans la même carrière d'utilité et d'instruction, et pour cette corporation de solliciter le secours du gouvernement, et d'encourager et favoriser par tous les moyens qui sont à sa disposition, un objet si désirable qu'un musée national et qui, est-on persuadé, n'a besoin que d'être commencé pour assurer un succès rapide et satisfaisant.

5. Que l'expérience des autres pays démontre que la création d'un musée national et la possession d'un édifice convenable pour le recevoir, ont déterminé des dons et des legs de collecteurs et de bienfaiteurs particuliers, excédant de beaucoup la valeur de l'argent public dépensé pour fonder et maintenir l'institution, et qu'il est désirable que le Canada profite de cette tendance à la générosité parmi les hommes de science.

6. Que conformément aux sentiments ci-dessus, une adresse renfermant ces vues, soit présentée par la corporation à Son excellence le comte d'Elgin, noble sur qui la science a des titres héréditaires, pour le prier de vouloir bien recommander à la législature provinciale de faire une appropriation pour commencer la fondation d'un musée national en Canada ${ }^{16}$.

Cette proposition reçut l'assentiment du Conseil de ville mais elle n'eut pas de lendemain immédiat ${ }^{17}$. En effet, la Galerie nationale du Canada ne vit le jour qu'en 1880 grâce à l'initiative du Marquis de Lorne, alors gouverneur général. De plus, cette institution fut établie non pas à Québec mais à Ottawa, nouvelle capitale du Canada depuis $1867^{18}$. En 1907, l'historien de l'art J. Purves Carter se disait déçu des collections de la Galerie nationale et se

16 Le Canadien, 23 mai 1853, 2.

17 Ibid., 25 mai 1853, 2.

18 Jean Sutherland Boggs, The National Gallery of Canada (Toronto, Oxford University Press, 1971), vii-viii. 
demandait si Ottawa avait été un bon choix pour son établissement ${ }^{19}$. Il affirmait trouver le Musée de l'Université Laval de Québec plus riche et plus intéressant même s'il ne s'agissait que d'une institution privée ${ }^{20}$.

L'Université Laval de Québec avait acheté en 1874 l'ancienne collection de peintures de Joseph Légaré. Le 28 janvier 1875, le journal L'Opinion publique écrivait que l'Université «possède actuellement la première galerie de peinture et le plus beau musée dans le continent ${ }^{21}$. En 1909, l' «Association des anciens élèves et gradués de l'Université Laval à Québec » devait reprendre à son compte un vœu déjà émis par $\mathrm{Carter}^{22}$ et qui rejoignait les préoccupations manifestées par un Joseph Légaré plus d'un demi-siècle auparavant:

Pour l'ASSOCIATION, elle aura atteint son but si elle peut ainsi éclairer le public, mettre d'accord toutes les bonnes volontés, afin que s'élève dans notre ville un MUSÉE NATIONAL, bâti suivant les données modernes, en sorte que nos trésors ne soient pas à la merci d'une conflagration toujours menaçante.

L'on piacera donc à i'abri d'une perte irréparable ces livres, ces toiles, ces estampes; ce sera l'œuvre des œuvres, celle qui aura contribué à transmettre intact, à nos successeurs, le plus opulent patrimoine du Nouveau Monde, héritage légué par des siècles de dévouement et dont la conservation nous incombe à aussi juste titre que celle de nos institutions et de notre langue $»^{23}$.

Le «Musée national» tel que souhaité par l'Association ne vit jamais le jour. Lors de l'ouverture du Musée du Québec en 1933, les collections de l'Université n'y furent pas intégrées.

19 Personnage controversé pour ses attributions audacieuses, Carter n'avait sans doute pas tort quant à l'institution elle-même puisque la fondation effective de la Galerie nationale du Canada n'eut lieu qu'en 1913. Voir Boggs, op. cit., vii.

20 [J. Purves Carter], A Shrine of Art. Many Noble Paintings Treasures which not many Canadians know their Country possesses (Québec, Laflamme et Proulx, 1907), 7-8. Ce texte rassemblait une série d'articles de Carter d'abord publiés dans le Quebec Chronicle et dans la Montreal Gazette. Il fut repris en 1908 en appendice du catalogue des peintures de l'Université Laval. Voir Carter, Descriptive and Historical Catalogue of the Paintings in the Gallery of Laval University, Quebec (Québec, L'Événement, 1908), 230 pages.

21 L'Opinion publique. Journal illustré (Montréal), 28 janvier 1875, 47. Le musée de l'Université Laval est aujourd'hui connu sous le nom de "Musée du Séminaire de Québec», lequel occupe des locaux de l'ancienne Université Laval.

22 En 1907. Voir Carter, A Shrine of Art, 61.

23 Carter, Musée de Peintures, Université Laval, Québec (Québec, "Telegraph», 1909), 22 pages. Préface de l'Association des anciens élèves et gradués de l'Université Laval à Québec, 3-4. 